\title{
First report of Acidovorax citrulli causing a leaf spot disease on tomato plants in Greece
}

\author{
Dimitris Malliarakis ${ }^{1}$ (D) Evaggelia Mpalantinaki ${ }^{1} \cdot$ Marianthi G. Pagoulatou $^{1} \cdot$ Konstantina Lorenzou $^{1} \cdot$ \\ Dimitrios E. Goumas ${ }^{1}$
}

Received: 2 September 2020 / Accepted: 6 October 2020 / Published online: 19 October 2020

(C) Società Italiana di Patologia Vegetale (S.I.Pa.V.) 2020

Keywords Tomato $\cdot$ Acidovorax citrulli $\cdot$ Detection $\cdot$ Greece $\cdot$ Europe

Necrotic black spots, often with chlorotic haloes, were observed on leaves of tomato seedlings growing in two transplant houses in Greece, during June 2019 and April 2020. Approximately 20 to $30 \%$ of the seedling plants were diseased, causing serious economic losses to the houses. Isolations from the infected leaf tissues on King's B medium consistently yielded a slow-growing and nonfluorescent bacterium, forming cream-coloured colonies. Recently, these isolates were characterized as Acidovorax citrulli (Ac) with the following methodologies. They were found to be gram-negative, aerobic, oxidase-positive and negative for arginine, aesculin, gelatin and levan tests. They also induced hypersensitive response on tobacco and reacted with specific antiserum for Ac (Cat. No. 07354/02, Loewe $囚$ ). Additionally, the isolates were identified by PCR amplification with specific primers SEQ ID no.3/ no.4 (Schaad et al. 2000). Blast analysis of $16 \mathrm{~S}$ rDNA sequence of the specific isolate HMU50556 (424 bp, GenBank Accession No. MT906357) showed 99.53\% similarity to Ac type strain ICMP7500 = ATCC29625 (NR 041758.1), strain T1 (WSBA01000009.1) and AAC00-1 (NZ RCCQ01000001.1). Furthermore, the gyrB partial sequence ( $87 \overline{9}$ bp, MT912472) was 99.54\% identical to Ac type strain ICMP7500 (GU385478.1) and $100 \%$ identical to strains T1 (NZ WSBA01000009.1) and AAC00-1 (NC_008752.1). Pathogenicity tests were conducted on tomato and watermelon seedlings by spray-inoculation with

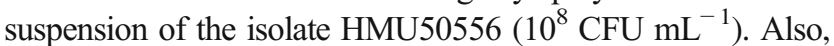

Electronic supplementary material The online version of this article (https://doi.org/10.1007/s42161-020-00677-1) contains supplementary material, which is available to authorized users.

Dimitris Malliarakis

dimmal@hmu.gr

1 Laboratory of Biological and Biotechnological Applications (Plant Bacteriology), Department of Agriculture, School of Agricultural Sciences, Hellenic Mediterranean University (HMU), Heraklion Crete 71004, Greece mature watermelon fruits were inoculated using toothpick technique. Symptoms were developed within 7 days and resembled those of natural infections. The bacterium was then re-isolated from infested tissues of all seedlings and identified as Ac, fulfilling Koch's postulates. To our knowledge, this is the first report of Ac causing disease on tomato seedlings in Greece, Europe, confirming previous findings in Israel (Chalupowicz et al. 2020) that this species can infect other plants than cucurbits. Our observations indicate possible transmission and spread of Ac through tomato seeds.

Acknowledgements This research has been co-financed by the European Union and Greek national funds through the Operational Program Competitiveness, Entrepreneurship and Innovation, under the call RESEARCH - CREATE - INNOVATE (project: VEG-PROTECT; code: T1EDK-04142).

Data availability Data presented in this Note has been submitted in the NCBI GenBank.

\section{Compliance with ethical standards}

Conflict of interest The authors declare no conflict of interest.

Consent for publication All authors have approved the manuscript and agree with its submission to Journal of Plant Pathology.

\section{References}

Chalupowicz L, Reuven M, Dror O, Sela N, Burdman S, Manulis-Sasson S (2020) Characterization of Acidovorax citrulli strains isolated from solanaceous plants. Plant Pathol. https://doi.org/10.1111/ppa.13239

Schaad NW, Song WY, Hatziloukas E (2000) PCR primers for detection of plant pathogenic species and subspecies of acidovorax. United States Department of Agriculture patents

Publisher's note Springer Nature remains neutral with regard to jurisdictional claims in published maps and institutional affiliations. 